\title{
Learning from Imperfect Data Weak Labels, Shifting Domains, and Small Datasets in Medical Imaging
}

\author{
Marleen de Bruijne ${ }^{1,2}$ \\ ${ }^{1}$ Erasmus MC, Rotterdam, The Netherlands \\ ${ }^{2}$ University of Copenhagen, Denmark \\ marleen.de.bruijne@gmail.com
}

Machine learning approaches, and especially deep neural networks, have had tremendous success in medical imaging in the past few years. Machine learningbased image reconstruction techniques are used to acquire high-resolution images at a much faster pace than before. Automated, quantitative image analysis with convolutional neural networks is as accurate as the assessment of an expert observer. Imaging biomarkers extracted via machine learning improve diagnosis, prognosis, and treatment decisions, and the first autonomous AI systems have been approved for diagnostic use and for patient triage in emergency radiology settings.

Machine learning however requires training datasets that are representative of the target data to analyze, cover the range of variation that will be observed in the target data, and are carefully labelled, often with time-consuming manual annotation strategies that require input from clinical experts. This hampers the adoption of machine learning in many medical image analysis tasks. In this talk, various approaches are discussed to make machine learning techniques work in practical situations, where training data is limited, data is highly heterogeneous, annotations are difficult to obtain or may be wrong, and training data may not be representative. Possible solutions include semi-supervised and weakly labeled learning, domain adaptation, and crowd-sourcing of visual analysis.

The potential of direct, machine learning-based diagnostics and prognostics is also discussed. Currently, most quantitative imaging biomarkers used for diagnosis and prognosis are factors that are already well-known to indicate disease. With such image quantification designed by experts-and AI models trained to mimic these experts-simplifications are made and the focus is on a small number of easily quantifiable image aspects. Machine learning enables a new, more data-driven approach. Image characteristics related to disease outcome can be learned directly from databases that combine medical imaging data with patient outcomes (e.g., the clinical diagnosis, therapy outcome, or future disease progression). This fully exploits the rich information present in medical imaging data and does not require time-consuming and error-prone manual annotations. This can result in stronger, more predictive imaging biomarkers, which is emphasized on applications in neuro-, pulmonary, and vascular imaging. 